\title{
Moving Vehicles Detection Based on Improved Gaussian Mixture Model
}

\author{
Y.L. Ma \\ Department of Automotive Engineering \\ Academy of Military Transportation \\ China
}

\author{
Z.C. Zhang \\ Department of Automotive Engineering \\ Academy of Military Transportation \\ China
}

\author{
Y.C. Li \\ Engineering Research Centre for Transportation Safety (Ministry of Education) \\ Wuhan University of Technology \\ China
}

\begin{abstract}
Vehicles detection has a great significance on traffic congestion relief, traffic accidents prevention and treatment. This paper proposes an improved Gaussianmixture model(GMM) to detect moving vehicles because GMM always detects background for foreground in complex environment. The new algorithm combines GMM with frame difference. Then, the whole vehicle can be detected by this new algorithm because of the enhanced demarcation point between background and foreground. Finally, the moving vehicles detection system is established. Experiments in different weather(sunny and rainy) and different time(day and night) have been made. They can prove that the new algorithm has been greatly improved in the aspects of adaptability, accuracy, real-time and so on. The movingvehicles can also be detected correctly and effectively in the situation with various complicated factors.
\end{abstract}

Keywords- transportation safety; vehicles detection; Gaussian mixture model (GMM); frame difference

\section{INTRODUCTION}

With the vehicle popularity and world urbanization, the numbers of vehicles increase rapidly not only in developed country but in developing country. Hence, vehicles detection has a great significance on traffic congestion relief, traffic accidents prevention and treatment[1]. There are several methods to detect vehicles: radar, GPS system, video technology and some hardware devices like vehicle inspection coil. However, it costs so much and difficulty maintenance for vehicle inspection coil while the terrain and complex analysis prevent the radar widely used. Meanwhile, there are some errors in GPS system, and the signal cannot cover some places like tunnel, the beneath of bridge. Then, video technology has some unique advantages: low cost, easy maintenance and simple calculation. What's more, HD camera can shoot moving vehicle clearly.

There are several common methods to detect the moving objects based on the video: optical follow[2], background subtraction[3], frame difference[4] and statistic model[5]. Background subtraction is the furthest and the most widely used method.
Hence, this paper proposes an improved algorithm of GMM to detect moving vehicles, which combines frame difference and GMM. This algorithm can increase the adaptability of road, vehicle detection accuracy and practicality.

The main contributions of this paper are summarized as follow: 1) select a place to collect the moving vehicle videos; 2) propose a new algorithm to improve GMM; 3) build a vehicle detection system.

The structure of this paper is organized as follows. Section 1 introduces the background and surveys the literatures. Section 2 proposes a new algorithm to improve GMM depended on GMM. Section 3 builds a vehicle detection system. Section 4 presents the experimental results with real data. Conclusions of this paper are drawn in Section 5.

\section{AN IMPROVED GMM}

This part main introduces an improved GMM, firstly GMM is analyzed, and it is ineffective in large and slow vehicles. When vehicles have a low velocity from quiescence to moving, the shadow appears easily. Meanwhile, GMM cannot solve the noises where in some complex scene like branches swinging.

Hence, an improved GMM is proposed to solve the problems above, it can increase the accuracy, timeliness and practicality.

\section{A. Introduction of GMM}

In GMM, every pixel is built in $\mathrm{K}$ GMM(K is usually taken from 3 to 5), hence, the sample of pixel $x_{t}$ in $t$ has a probability density function, the function is represented by the $K$ probability density functions' weighted sum:

$$
P\left(x_{t}\right)=\sum_{i=1}^{K} \omega_{i, t} \eta_{i, t}\left(x_{t}, \mu_{i, t}, \sum_{i, t}\right)(1)
$$


Where $K$ is the number of Gaussian model; $\omega_{i, t}$ is Gaussian model weights, hence, the sum of $K$ weights is 1 ; $\mu_{i, t}$ is $i^{\text {th }}$ mean of Gaussian distribution; $\sum_{i, t}$ is covariance matrix. It can assume that RGB itself is independent with each other, so $\sum_{i, t}=\sigma_{i, t}^{2} I$ ( $\sigma_{i, t}^{2} I$ is the $i^{\text {th }}$ Gaussian model variance in time $t$ ) is the $i^{\text {th }}$ Gaussian distribution in time $t$, it is given as follow:

$$
\eta_{i, t}\left(x_{t}, \mu_{i, t}, \sum_{i, t}\right)=\frac{1}{(2 \pi)^{\frac{n}{2}}\left|\sum_{i, t}\right|^{1 / 2}} e^{-\frac{1}{2}\left(x_{t}-\mu_{i, t}\right)^{T \square \Sigma^{-1}\left(x_{t}-\mu_{i, t}\right)}}
$$

Where $n$ is the dimension of $x_{t}$.

The method of GMM can be given as follows:

Step 1: the every pixel of current frame can be got, it is matched with $K$ Gaussian distribution which has existed, $\left|x_{t}-\mu_{i, t-1}(x, y)\right| \leq 2.5 \sigma_{i, t-1}$ is matching condition.

Step 2: if the matching conditions are met, the pixel values and Gaussian distribution has a success match, otherwise:

1) While $k<K$, new Gaussian distribution should be increased.

2) While $k=K$, new Gaussian distribution is instead of the priority minimum distribution of $\sigma_{i, t}^{2}=\frac{\omega_{i, t}}{\left|\sum_{i, t}\right|}$.

$x_{t}$ is the mean of new distribution, and it has a big variance initial (which is 50), the weight is a smaller value.

Step 3: the mean and standard deviation unmatched keep unchanged, if the parameters of the $i^{\text {th }}$ Gaussian distribution is matched, it can update as follows:

$$
\begin{gathered}
\mu_{i, t}=(1-\theta) \mu_{i, t-1}+\theta x_{i, t} \\
\sigma^{2}{ }_{i, t}=(1-\theta) \sigma^{2}{ }_{i, t-1}+\theta \square\left(x_{i, t}-\mu_{i, t-1}\right)^{T}\left(x_{i, t}-\mu_{i, t-1}\right) \\
\sum_{i, t}=(1-\theta) \sum_{i, t-1}+\theta \times \operatorname{diag}\left[\left(x_{i, t}-\mu_{i, t-1}\right)^{T}\left(x_{i, t}-\mu_{i, t-1}\right)\right] \\
\theta=\varepsilon \eta\left(x_{i, t} \mid \mu_{i, t}, \sum_{i, t}\right)
\end{gathered}
$$

Where $\varepsilon$ is the learning rate, $0 \leqslant \varepsilon \leqslant 1$, it decides the update speed of the background; $\varepsilon \theta$ is the parameter learning rate.

Step 4: when the match inspection finished, eqn(7) can update the every Gaussian distribution's weight of the same pixel:

$$
\omega_{i, t}=(1-\varepsilon) \omega_{i, t-1}+\varepsilon \sqcap G_{i, t}
$$

If the $i^{\text {th }}$ Gaussian distribution match with $x_{i, t}, G_{i, t}=1$, otherwise, $G_{i, t}=0$. It is normalized after all of the Gaussian distribution's weight finishes update.
Step 5: the Gaussian model finishes update, $K$ Gaussian distribution of every pixel can be described as follow, and it obeys in front of the $B$ Gaussian distribution which are arrayed by priority $\varphi_{i, t}=\omega_{i, t} /\left|\sum_{i, t}\right|$ :

$$
B=\arg \min \left(\sum_{k=1}^{b} \omega_{k}>T_{1}\right) ; 0.5<T_{1}<1
$$

The matching inspection is continuous by $x_{i, t}$ and $B$ Gaussian distribution, if $x_{i, t}$ can match with any points of the previous $B$ Gaussian distribution, this pixel is a background point; otherwise, it is a foreground point, then finishes the moving object detection.

\section{B. Improved Gaussian Mixed Modeling}

1) Improved frame difference. Frame difference has various advantages such as simple principle, simple program design, and rapid computation and so on. However, through some tests, the frame difference also has many drawbacks such as part of moving objects detected only, dispersion object pixel, hole inside. Hence, this part proposes an improved frame difference; it conducts to 8 neighborhoods of the pixel. It can be given as follows:

Expression of two frame difference is:

$$
D_{i}(x, y)=\left|f_{i}(x, y)-f_{i-1}(x, y)\right|
$$

Where $f_{i}(x, y)$ is the grey level of the current frame image, $f_{i-1}(x, y)$ is the grey level of the previous frame image. The pixel $f(x, y)$ 's 8 neighborhoods are conducted as follow figure:

\begin{tabular}{|l|l|l|}
\hline$f(x-1, y+1)$ & $f(x, y+1)$ & $f(x+1, y+1)$ \\
\hline$f(x-1, y)$ & $f(x, y)$ & $f(x+1, y)$ \\
\hline$f(x-1, y-1)$ & $f(x, y-1)$ & $f(x+1, y-1)$ \\
\hline \multicolumn{3}{|c}{$f(x, y)$,s 8 NEIGHBORHOODS }
\end{tabular}

Hence, the 8 neighborhoods sum of $D_{i}(x, y)$ is:

$$
\begin{aligned}
& \sum_{i}(x, y)=D_{i}(x-1, y+1)+D_{i}(x, y+1)+D_{i}(x+1, y+1) \\
& +D_{i}(x-1, y)+D_{i}(x+1, y)+D_{i}(x-1, y-1)+D_{i}(x, y-1)+D_{i}(x+1, y-1)
\end{aligned}
$$

The expression of the improved frame difference is given:

$$
\eta_{i}(x, y)=\left\{\begin{array}{l}
1, D_{i}(x, y)>T_{2} \cap \sum_{i}(x, y)>T_{3} \\
0, \text { others }
\end{array}\right.
$$

Where $T_{2}$ is 0.01 , because it prevents the current frame background to sentence for moving objects; $T_{3}$ is the 8 neighborhoods threshold of second-order differential image, and it equals to 0.07 .

The improved frame difference can get the whole moving objects, which pixel distribution is concentrate. What's more, the computation and complexion is also simple. 
2) GMM combines with improved frame difference.GMM combines with the improved frame difference, and the specific process is as follows:

Step 1:

$$
\eta_{i}(x, y)=\left\{\begin{array}{l}
A_{c}, \quad D_{i}(x, y)>T_{2} \cap \sum_{i}(x, y)>T_{3} \\
A_{b g}, \text { others }
\end{array}\right.
$$

Where $A_{c}$ is the changed zone between two frames, and it can be detected by improved frame difference. This zone includes $A_{u}$ (background reveal zone in precious frame) and $A_{m}$ (background reveal zone in current frame)

Step 2: the changed zone which has distinguished is $A_{c}$, each pixel of $A_{c}$ matches with $B$ Gaussian distribution which is arrayed as priority from big to small. The matching condition satisfies $\left|x_{t}(x, y)-\mu_{i, t-1}(x, y)\right| \leq 2.5 \sigma_{i, t-1}$. It can classify with any pixels matching:

$$
\left\{\begin{array}{l}
\left|x_{t}(x, y)-\mu_{i, t-1}(x, y)\right| \leq 2.5 \sigma_{i, t-1}, x_{t}(x, y) \in A_{u} \\
\left|x_{t}(x, y)-\mu_{i, t-1}(x, y)\right|>2.5 \sigma_{i, t-1}, x_{t}(x, y) \in A_{m}
\end{array}\right.
$$

Where $0<i \leq B, \mu_{i, t-1}(x, y), \sigma_{i, t-1}$ are the mean and variance of the $i^{\text {th }}$ Gaussian distribution in time $t-1$. If the conditions are matching, it belongs to $A_{u}$, otherwise, it belongs to $A_{m}$.

GMM is combined with the improved frame difference, first of all, the whole vehicle can be extracted by the frame difference, the problem which GMM cannot detected slow and large vehicles is solved well. In addition, the improved frame difference is used in the early GMM, the threshold of background and foreground can be selected effectively.

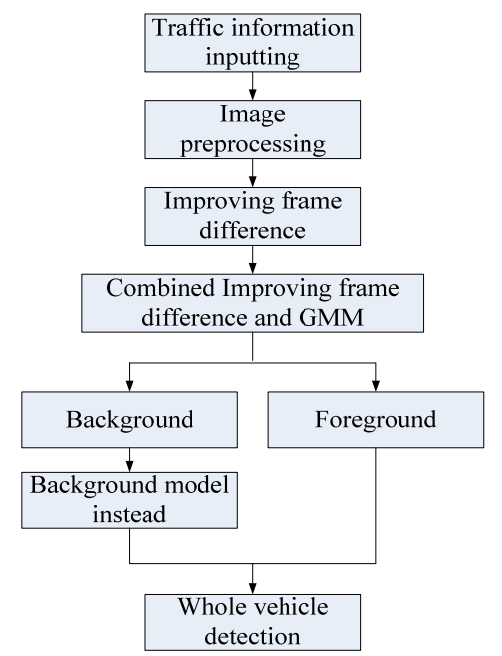

FIGURE II. FLOWCHART OF VEHICLE DETECTION SYSTEM.

\section{VEHICLE DETECTION SYSTEM}

This system is used by opencv1.0. The development platform is VC6.0. The main algorithm is the improved
GMM. The flowchart shows as fig.2. Fig.3 is the moving vehicle detection system.

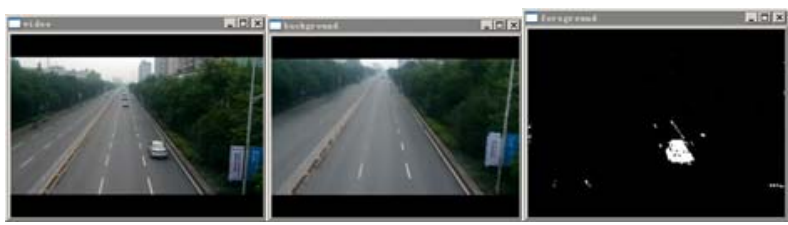

(a) the original image (b) background image (c) the object image FIGURE III. MOVING VEHICLE DETECTION SYSTEM.

\section{EXPERIMENTAL RESULTS}

The video camera used is a Sony HDR-PJ1790. We select the second bridge of Wuhan University of Technology(WUT) in Wuhan, Youyi Avenue as collection location. Youyi Avenue is an important transportation hub in Wuhan with six lanes, rich vehicle models and large traffic flow. In order to enrich the collect samples, different weather like sunny rainy are selected. The collection time is from 8: 00 22: 00, the other time has less traffic flow, little environment difference from collection time. Hence, there is nothing to do in these times.

This part evaluates the algorithm, first of all, driving vehicles are evaluated by GMM and improved GMM, then the results are compared; secondly, the improved GMM is detected in different environment.

In order to evaluate the improved GMM, three different times are selected to detect vehicles, we can get from fig. 4 to fig.6 that the improved GMM can separate the background and foreground accurately. The figures time from 4 to 6 are sunny in the morning, rainy in the afternoon and sunny in the evening, respectively, two frame figures are extracted from every video, the two figures record the vehicles from bridge blew to far from the bridge. Where, figure (a) and (d) represent the original images, figure (b) and (d) are the images detected by GMM, figure (c) and (f) are the images detected by the improved GMM. After compared, we can see that, both background and foreground are easy confusion by GMM, so that many details are in the object images; the whole vehicles can be detected and the noise of the foreground is filtered well by the improved GMM.

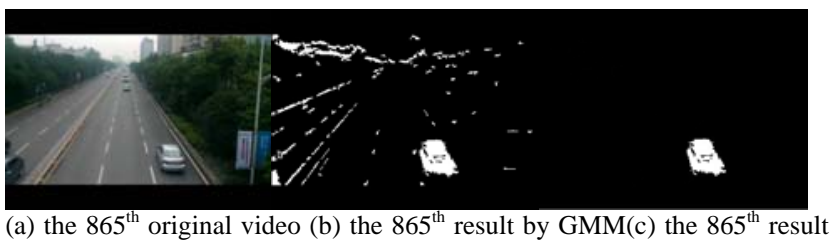

by the improved GMM

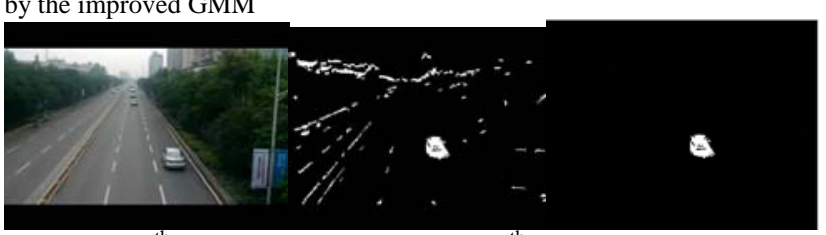

(d) the $880^{\text {th }}$ original video (e) the $880^{\text {th }}$ result by GMM (f) the $880^{\text {th }}$ result by the improved GMM

FIGURE IV. RESULTS OF GMM AND THE IMPROVED GMM IN SUNNY. 


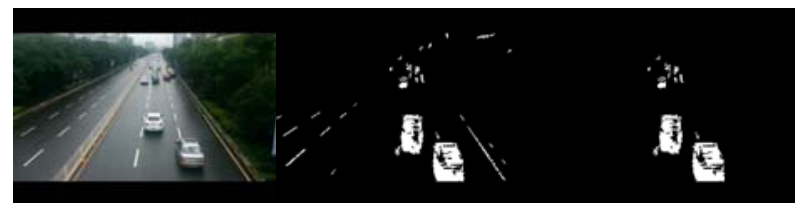

(a) the $394^{\text {th }}$ original video (b) the $394^{\text {th }}$ result by GMM (c) the $394^{\text {th }}$ result by the improved GMM

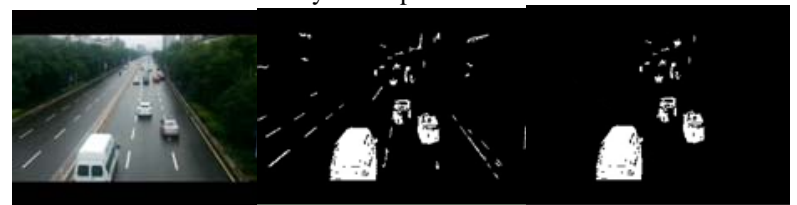

(d) the $408^{\text {th }}$ original video (e) the $408^{\text {th }}$ result by GMM (f) the $408^{\text {th }}$ result by the improved GMM

FIGURE V. RESULTS OF GMM AND THE IMPROVED GMM IN RAINY.

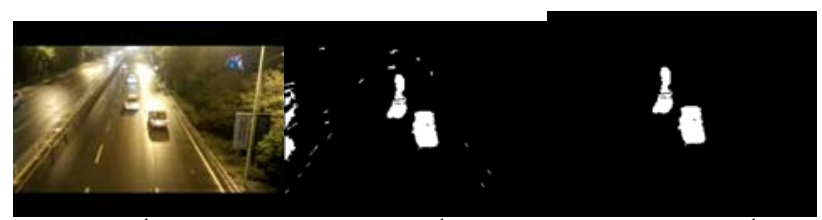

(a) the $634^{\text {th }}$ original video (b) the $634^{\text {th }}$ result by GMM (c) the $634^{\text {th }}$ result by the improved GMM

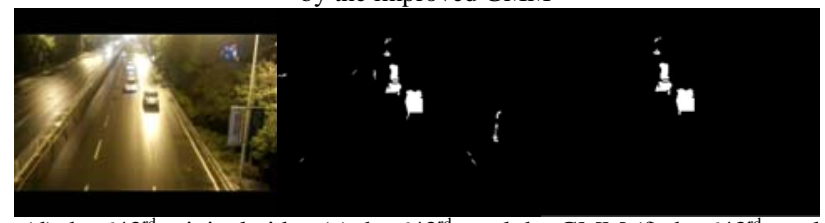

(d) the $643^{\text {rd }}$ original video (e) the $643^{\text {rd }}$ result by GMM (f) the $643^{\text {rd }}$ result by the improved GMM

FIGURE VI. RESULTS OF GMM AND THE IMPROVED GMM IN EVENING.

\section{CONCLUSIONS}

This paper proposes a new GMM algorithm combined with frame difference and GMM, it aims that the background and foreground are easy confusion by GMM. This algorithm enhances the contrast between the background and foreground; the vehicle can be detected from the original image effectively. Finally, a vehicle detection system is built by this algorithm. This system can detect moving vehicles in different weather(sunny and rainy) by experiments. It has a great significance on traffic congestion relief, traffic accidents prevention and treatment.

\section{ACKNOWLEDGEMENT}

This study is supported by Nation Natural Science Foundations of China (No. 91120306 and No. 61174173), China Postdoctoral Science Foundation (2013M542433), and Tianjin Natural Science Foundation (14JCQNJC01600).

\section{REFERENCE}

[1] Li, K. M. \& Zhang, Q., Review of Ground Vehicles Recognition. Acta Electronica Sinica, pp. 538-546, 2014.

[2] Hu, W. M., Tan, T. N. \& Wang L., A Survey on Visual Surveillance of Object Motion and Behaviors. IEEE Transactions on Systems, Man and Cybernetics, 4(3), pp. 334-352, 2004.

[3] Cheng, S. H.\& Hu, C. H, Automatic Segmentation Algorithm Based on Spatio-temporal Domain for Video Objects. Journal of Applied Optics, pp. 768-771, 2009.
[4] Dickinson P, Hunter A \& Appiah K, A Spatially Distributed Model for Foreground Segmentation. Image and Vision Computing, 27(9), pp. 1326-1335, 2009.

[5] Chang, X. F., Zhang, W. S. \& Dong, W. S., Multi-species Mixture Gaussian Background Model Based on Visual Characteristics. Chinese Journal of Image and Graphics, 16(5), pp. 829-834, 2011. 